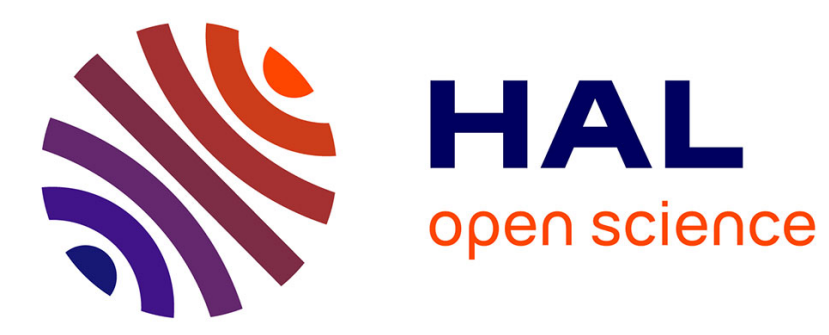

\title{
Project Games
}

Vittorio Bilò, Laurent Gourvès, Jérôme Monnot

\section{To cite this version:}

Vittorio Bilò, Laurent Gourvès, Jérôme Monnot. Project Games. Algorithms and Complexity - 11th International Conference, CIAC, May 2019, Rome, Italy. 10.1007/978-3-030-17402-6 . hal-02343786

\section{HAL Id: hal-02343786 https://hal.science/hal-02343786}

Submitted on 3 Nov 2019

HAL is a multi-disciplinary open access archive for the deposit and dissemination of scientific research documents, whether they are published or not. The documents may come from teaching and research institutions in France or abroad, or from public or private research centers.
L'archive ouverte pluridisciplinaire HAL, est destinée au dépôt et à la diffusion de documents scientifiques de niveau recherche, publiés ou non, émanant des établissements d'enseignement et de recherche français ou étrangers, des laboratoires publics ou privés. 


\title{
Project Games ${ }^{\star}$
}

\author{
Vittorio Bilò $^{1}$, Laurent Gourvès ${ }^{2}$, and Jérôme Monnot ${ }^{2}$ \\ 1 Department of Mathematics and Physics "Ennio De Giorgi", University of Salento, \\ Lecce, Italy, vittorio.bilo@unisalento.it \\ ${ }^{2}$ CNRS, Université Paris-Dauphine, Université PSL, LAMSADE, 75016 Paris, \\ France, \{laurent.gourves, jerome.monnot\}@dauphine.fr
}

\begin{abstract}
We consider a strategic game called project game where each agent has to choose a project among his own list of available projects. The model includes positive weights expressing the capacity of a given agent to contribute to a given project The realization of a project produces some reward that has to be allocated to the agents. The reward of a realized project is fully allocated to its contributors, according to a simple proportional rule. Existence and computational complexity of pure Nash equilibria is addressed and their efficiency is investigated according to both the utilitarian and the egalitarian social function.
\end{abstract}

Keywords: Strategic Games, Price of Anarchy/Stability, Congestion

\section{Introduction}

We introduce and study the PROJECT GAME, a model where some agents take part to some projects. Every agent chooses a single project but several agents can select the same project. This situation happens for example when some scientists decide on which problem they work, when some investors choose the business in which they spend their money, when some benefactors select which artistic project they support, etc. Our model includes positive weights which express the capacity of a given agent to contribute to a given project. By assumption, a project is realized if it is selected by at least one agent. The realization of a project produces some reward that has to be allocated to the agents.

We take a game theoretic perspective, i.e. an agent's strategy is to select, within the projects that are available to her, the one inducing the largest piece of reward. Therefore, the way the rewards are allocated is essential to this game. Here we suppose that the reward of a realized project is fully allocated to its contributors, according to a simple proportional rule based on the aforementioned weights.

Our motivation is to analyze the impact of this simple and natural allocation rule. Do the players reach a Nash equilibrium, that is a stable state in which no one wants to deviate from the project she is currently contributing? How bad is a Nash equilibrium compared to the situation where a central authority would,

\footnotetext{
* Supported by ANR Project CoCoRICo-CoDec
} 
at best, decide by which agent(s) a project is conducted? In other words, does the allocation rule incentivize the players to realize projects that optimize the total rewards?

The Model. The PROJect GAME is a strategic game with a set of $n$ players $N=\{1, \cdots, n\}=[n]$ and a set of $m$ projects $M=\{1, \cdots, m\}=[m]$. The strategy space of every player $i$, denoted by $S_{i}$, is a subset of $M$. We assume that $\bigcup_{i \in N} S_{i}=M$ and a strategy for player $i$ is to select a project $j \in S_{i}$. Each project $j \in M$ has a positive reward $r_{j}$. We suppose without loss of generality that the minimum reward is always equal to 1 . Each player $i \in N$ has a positive weight $w_{i, j}$ when she selects project $j$.

The load of project $j$ under strategy profile $\sigma$, denoted by $L(\sigma, j)$, is the total weight of the players who play $j$. Thus, $L(\sigma, j)=\sum_{\left\{i \in N: \sigma_{i}=j\right\}} w_{i, j}$.

The utility of player $i$ (that she wants to maximize) under $\sigma$ is defined as

$$
u_{i}(\sigma)=\frac{w_{i, \sigma_{i}}}{L\left(\sigma, \sigma_{i}\right)} r_{\sigma_{i}}
$$

A player's utility is defined as a portion of the reward of the realized project that she is contributing to. This portion is proportional to the player's weight.

We will sometimes consider special cases of the PROJECT GAME. An instance of the PROJECT GAME is symmetric when $S_{i}=M$ for every player $i$. The players' weights are universal when, for every player $i, w_{i, j}$ is equal to some positive number $w_{i}$ for every project $j$; in particular, they are identical when $w_{i}=1$ for every player $i$. The weights are project-specific when they are not universal. The projects' rewards are identical when the reward is the same for all projects, and this reward is equal to 1 by assumption.

A strategy profile $\sigma$ is a pure Nash equilibrium if for each $i \in N$ and $j \in S_{i}$, $u_{i}(\sigma) \geq u_{i}\left(\sigma_{-i}, j\right)$ where $\sigma^{\prime}=\left(\sigma_{-i}, j\right)=$ is defined by $\sigma_{\ell}^{\prime}=\sigma_{\ell}$ for $\ell \in N \backslash\{i\}$ and $\sigma_{i}^{\prime}=j$. For a Project game $G$, denote by $\operatorname{NE}(G)$ its set of pure Nash equilibria.

For a strategy profile $\sigma, P(\sigma)=\{j \in M: L(\sigma, j)>0\}$ will denote the set of projects selected by some players in $\sigma$. The social utility under strategy profile $\sigma$, denoted by $\mathrm{U}(\sigma)$, is defined as the total sum of the rewards of the selected projects (also known as the utilitarian social welfare), i.e., $\mathrm{U}(\sigma)=\sum_{j \in P(\sigma)} r_{j}$. Note that $\mathrm{U}(\sigma)=\sum_{i \in N} u_{i}(\sigma)$. A social optimum, denoted as $\sigma^{*}$, is a strategy profile maximizing $\mathrm{U}$.

Given a PROJECT GAME $G$, the price of anarchy of $G$ is the worst-case ratio between the social utility of a social optimum and the social utility of a pure Nash equilibrium for $G$, namely, $\operatorname{PoA}(G)=\sup _{\sigma \in \mathrm{NE}(G)} \frac{\mathrm{U}\left(\sigma^{*}\right)}{\mathrm{U}(\sigma)}[1$; the price of stability of $G$ is the best-case ratio between the social utility of a social optimum and the social utility of a pure Nash equilibrium for $G$, namely, $\operatorname{PoS}(G)=\inf _{\sigma \in \mathrm{NE}(G)} \frac{\mathrm{U}\left(\sigma^{*}\right)}{\mathrm{U}(\sigma)}[2]$.

For any two integers $n, m>1$, let $\mathcal{G}_{n, m}$ denote the set of all PROJECT GAMES with $n$ players and $m$ projects. We define $\operatorname{PoA}(n, m)=\sup _{G \in \mathcal{G}_{n, m}} \operatorname{PoA}(G)$ (resp. 
$\left.\operatorname{PoS}(n, m)=\sup _{G \in \mathcal{G}_{n, m}} \operatorname{PoS}(G)\right)$ as the price of anarchy (resp. stability) of games with $n$ players and $m$ projects.

Our Contribution. We focus on existence, computational complexity and efficiency of pure Nash equilibria in PROJECT GAMEs. Given the structural simplicity of these games, it will be possible to derive some results from the state of the art of similar classes of games.

For instance, by making use of the notion of better response equivalence 3, we derive that the problem of computing a pure Nash equilibrium in the PROJECT GAME with universal weights belongs to the complexity class PLS and can be solved in polynomial time as long as at least one of the following three conditions holds: the game is symmetric, the rewards are identical, the weights are identical. For the more general case of project-specific weights, instead, we show by means of a potential function argument that the problem is in PLS as long as the rewards are identical. Without this assumption, the problem gets fairly much more complicated and even the existence of pure Nash equilibria remains an open problem.

As to the efficiency of pure Nash equilibria, it is easy to see that the PROJECT GAMEs belong to the class of valid utility games. For these games, Vetta 4 gives an upper bound of 2 on the price of anarchy. We show that this bound is tight only for the case of asymmetric games with non-identical rewards and non-identical weights. In all other cases, we give refined bounds parameterized by both the number of players and projects, also with respect to the price of stability. All these bounds are shown to be tight except for one case involving the price of anarchy of asymmetric games with identical rewards and identical weights. For this particular variant of the game, we also consider an interesting restriction in which all players have exactly two available strategies. These games admit a multigraph representation and we provide some bounds on the price of anarchy as a function of the multigraph topology.

Before concluding, we explore the efficiency of equilibria under an alternative notion of social welfare which focuses on the utility of the poorest player. In this document, some proofs are omitted due to space constraints but they will appear in a journal version.

Related Work. Our PROJECT GAMEs fall within the class of monotone valid utility games introduced by Vetta [4] and further considered in [5|6|7/8|9|10|11]. In a monotone valid utility game there is a ground set of objects $V$ and a strategy for a player consists in selecting some subset of $V$. A social function $\gamma: 2^{V} \mapsto \mathrm{R}$ associates a non-negative value to each strategy profile; $\gamma$ is assumed to be monotone and submodular. The utility of player $i$ in a strategy profile $\sigma$ is at least the value $\gamma(\sigma)-\gamma\left(\sigma_{-i}\right)$. Moreover, the sum of the players' utilities in $\sigma$ does not exceed the value $\gamma(\sigma)$. Vetta [4] shows that the price of anarchy of these games is at most 2 .

Among the special cases of monotone valid utility games considered in the literature, the one that mostly relates to our PROJECT GAMEs is the one studied 
by Kleinberg and Orel in [9. They consider a set of projects modeling open problems in scientific research and a set of players/scientists each of which chooses a single problem to work on. However, there are several differences between the two models which make the achieved results non comparable. In fact, in the games studied by Kleinberg and Orel, players may fail in solving a problem, and so the reward associated with each project is not always guaranteed to be realized; moreover, when a problem is solved, its reward is always shared equally among the solving players. This assumption makes these games instances of congestion games, whereas this is not the case in our PROJECT GAMEs.

Congestion games [12] is a well known category of strategic games which, by a potential argument [13, always admit a pure Nash equilibrium. In a congestion game, there is a set of resources $M$ and every players' strategy set is a non-empty subset of $2^{M}$. For example, $M$ contains the links of a network from which each player wants to choose a path. Each resource $j$ is endowed with a latency function $\ell_{j}$ which depends on the number of players having $j$ in their strategy. A player's cost is the sum of the latencies of the resources that she uses. This model received a lot of attention in the computer science community, see e.g. 14]. Congestion games where generalized to the case where the players have different weights (weighted congestion games), or when a resource's latency depends on the identity of the player (player specific congestion games) [15]. These extensions still admit a pure Nash equilibrium if the players' strategies are singletons. Nevertheless, a pure Nash equilibrium is not guaranteed when we combine weights and player-specific costs, even with singleton strategies [15]. Singleton congestion games with weighted players are also known as Load Balancing games (c.f. [16]): resources and players may represent machines and jobs, respectively. In this context each job goes on the machine that offers her the lowest completion time.

Finally, it is worth mentioning the PROJECT GAME is remotely connected with hedonic games [17] and the group activity selection problem [18, as the realized projects induce a partition of the player set.

\section{Existence of a Pure Strategy Nash Equilibrium}

In this section, we focus on the existence and efficient computation of pure Nash equilibria in the PROJECT GAME. We shall show how several positive results can be obtained from the realm of load balancing games and singleton congestion games by making use of the notion of better response equivalence [3]. Intuitively, two games are better response equivalent when, for every pair of strategies, they agree when one is better than the other (i.e., they have the same Nash dynamics graph). By definition, two games which are better response equivalent share the same set of pure Nash equilibria. Thus, existential and computational results for one game can be directly applied to the other. 
Fix a PROJECT GAME with universal weights. By (1), we have that, for each strategy profile $\sigma$, player $i \in N$ and strategy $j \in S_{i}$,

$$
u_{i}\left(\sigma_{-i}, j\right)>u_{i}(\sigma) \Longleftrightarrow \frac{L(\sigma, j)+w_{i}}{r_{j}}<\frac{L\left(\sigma, \sigma_{i}\right)}{r_{\sigma_{i}}} .
$$

If one interprets the set of projects as a set of related machines, where machine $j$ has a speed $r_{j}$, and the set of players as a set of tasks, where task $i$ has a processing time $w_{i}$, it follows immediately from (2) that any PROJECT GAME with universal weights is better response equivalent to a load balancing game with related machines. Similarly, a PROJECT GAME with universal weights and identical projects is better response equivalent to a load balancing game with identical machines and a PROJECT GAME with identical weights is better response equivalent to a singleton congestion game with linear latency functions.

So, for the PROJECT GAME with universal weights, the existential result for load balancing games with related machines as well as the polynomial time algorithm for the case of symmetric games, both given in [19], can be reused. For asymmetric games with identical rewards, the polynomial time algorithm given in 20] can be applied. For asymmetric games with identical weights, the algorithm given in 14] can be applied. These results are summarized in the following theorem.

Theorem 1 ([1920,14]). The PROJECT GAME with universal weights admits a potential function. Moreover, a pure Nash equilibrium can be computed in polynomial time when at least one of the following conditions is true: the game is symmetric, the rewards are identical, the weights are identical.

For the case of project-specific weights, no transformation to other known classes of games are possible (up to our knowledge) and a direct approach needs to be developed. For projects with identical rewards, we show the existence of pure Nash equilibria by providing a potential function argument.

Theorem 2. For the PROJECT GAME with identical rewards, the vector $\langle|P(\sigma)|$, $\Phi(\sigma)\rangle$, where $\Phi(\sigma):=\Pi_{j \in P(\sigma)} L(\sigma, j)$ lexicographically increases after every profitable unilateral deviation.

It follows from Theorem 2 that the better response dynamics of the PROJECT GAME with identical rewards never cycles: it always converges to a pure Nash equilibrium. As the potential function given in Theorem 2, as well as the one given in 19 for games with universal weights, can be computed in polynomial time, it follows that the problem of computing a pure Nash equilibrium in games with project-specific weights and identical rewards and in games with universal weights belongs to the complexity class PLS, see, for instance, 21.

For the case of general rewards and project-specific weights, it is easy to see that the PROJECT GAME is better response equivalent to a particular subclass of singleton weighted congestion games with player-specific linear latency functions and resource-specific weights. These games are defined as follows. There is a set of 
$n$ players $N=\{1, \cdots, n\}=[n]$ and a set of $m$ resources $R=\{1, \cdots, m\}=[m]$. Each player $i \in N$ can choose a resource from a prescribed set $S_{i} \subseteq R$ and has a weight $w_{i, j}>0$ on resource $j \in R$. The load (congestion) of resource $j$ in a strategy profile $\sigma$ is $L(\sigma, j)=\sum_{i \in N: \sigma_{i}=j} w_{i, j}$. Each resource $j \in R$ has a player-specific linear latency function $\ell_{j}^{i}(x)=\alpha_{j}^{i} x$, with $\alpha_{j}^{i} \geq 0$, for each $i \in N$. The cost of player $i$ in $\sigma$ is defined as $c_{i}(\sigma)=\ell_{\sigma_{i}}^{i}\left(L\left(\sigma, \sigma_{i}\right)\right)=\alpha_{\sigma_{i}}^{i} L\left(\sigma, \sigma_{i}\right)$.

To the best of our knowledge, singleton weighted congestion games with player-specific linear latency functions and resource-specific weights have been considered so far in the literature only under the assumption that the players' weights are not resource-specific, i.e., each player $i \in N$ has a weight $w_{i}>0$ for each resource $j \in S_{i}$. These games have been considered in 2223. In particular, 22 shows that they do admit a potential function if and only if $n=2$, while 23 . proves the existence of a pure Nash equilibrium for the cases of either $n=3$ or $m=2$; in the latter, a polynomial time algorithm for computing an equilibrium is also provided. However, there is no relationship between these games and our PROJECT GAMES. In fact, if from one perspective PROJECT GAMES are more general than singleton weighted congestion games with player-specific linear latency functions in the definition of the players' weights (which are resource-specific in the former and resource-independent in the latter), on the other hand singleton weighted congestion games with player-specific linear latency functions are more general than PROJECT GAMES in the definition of the latency functions (which are arbitrary in the former and resource-related in the latter).

We close this section with the most general case of the PROJECT GAME, but for a small number of players.

Proposition 1. The best response dynamics of the PROJECT GAME with two players always converges.

Proposition 2. The PROJECT GAME with three players always admits a pure Nash equilibrium.

\section{Social Utility and the Price of Anarchy/Stability}

In this section, we analyze the quality of pure Nash equilibria in the PROJECT GAME in term of price of anarchy and stability. Before presenting our complete characterization of their bounds, note that a social optimum can be computed efficiently.

Proposition 3. Maximizing the utilitarian social welfare of the PROJECT GAME can be done in polynomial time.

\subsection{Games with Identical Rewards}

In this subsection, we give results for games with identical rewards. The first result states that there is always a pure Nash equilibrium that is socially optimal. 
Theorem 3. For any two integers $n, m>1, \operatorname{PoS}(n, m)=1$.

Next, we show that, under the assumption of symmetric games, all pure Nash equilibria are socially optimal.

Theorem 4. For any two integers $n, m>1, \operatorname{PoA}(n, m)=1$ for symmetric games.

For asymmetric games, instead, next theorem shows that the price of anarchy rises to almost 2 even when considering universal weights.

Theorem 5. For any two integers $n, m>1$ and $s:=\min (n, m), \operatorname{PoA}(n, m) \geq$ $\frac{2\left\lfloor\frac{s-1}{2}\right\rfloor+1}{\left\lfloor\frac{s-1}{2}\right\rfloor+1}$ for games with universal weights.

A matching upper bound, which holds for the more general case of projectspecific weights is achieved in the following theorem.

Theorem 6. For any two integers $n, m>1$ and $s:=\min (n, m), \operatorname{PoA}(n, m) \leq$ $\frac{2\left\lfloor\frac{s-1}{2}\right\rfloor+1}{\left\lfloor\frac{s-1}{2}\right\rfloor+1}$.

By Theorems 5 and 6 , we get that $\operatorname{PoA}(n, m)=\frac{2\left\lfloor\frac{s-1}{2}\right\rfloor+1}{\left[\frac{s-1}{2}\right\rfloor+1}$ for games with both project-specific and universal weights.

Identical weights Here, we consider the case of games with identical weights. Games with this property admit an interesting representation via hypergraphs (it becomes multigraphs when $\left|S_{i}\right| \leq 2$ for each $i \in N$ ). A complete proof is given in Appendix.

Theorem 7. For identical weights and identical rewards and for any two integers $n>5, m>1,1.582 \approx \frac{e}{e-1} \leq \operatorname{PoA}(n, m) \leq \frac{5}{3} \approx 1.667$.

\subsection{Games with Non Identical Rewards}

In this subsection, we address the more general case of general rewards. We start by showing a lower bound on the price of stability which holds even for symmetric games with identical weights.

Proposition 4. For any two integers $n, m>1, \operatorname{PoS}(n, m) \geq 1+\frac{\min (n, m)-1}{n}$ for symmetric games with identical weights.

Proof. For any two integers $n, m>1$, consider a game with $n$ players of weight 1 , one project $p$ with reward $n+\epsilon$, where $\epsilon>0$ is an arbitrary number, and $m-1$ projects with reward 1 .

As choosing project $p$ is a dominant strategy for each player, this game has only one pure Nash equilibrium in which all the players select $p$. Under this strategy profile, the social utility is $n+\epsilon$. In a social optimum, a maximum number of $\min (n, m)$ projects can be selected by some player, so that the social utility is at most $n+\epsilon+\min (n, m)-1$. Thus, by the arbitrariness of $\epsilon$, the price of stability is at least $1+\frac{\min (n, m)-1}{n}$. 
We now show a matching upper bound that holds even for the price of anarchy of symmetric games with project-specific weights.

Theorem 8. For any two integers $n, m>1, \operatorname{PoA}(n, m) \leq 1+\frac{\min (n, m)-1}{n}$ for symmetric games with project-specific weights.

We now move to the case of asymmetric games. Again, we shall prove a lower bound on the price of stability which holds for universal weights and then provides a matching upper bound on the price of anarchy for the case of projectspecific weights. As to the upper bound, from Vetta's result [4], we have that, for any two integers $n, m>1, \operatorname{PoA}(n, m) \leq 2$ for games with project-specific weights. Now, we show the matching lower bound.

Proposition 5. For any two integers $n, m>1, \operatorname{PoS}(n, m) \geq 2$ for games with universal weights.

The lower bound for the price of stability given in Proposition 5 does not apply to games with identical weights. This leaves open the possibility to obtain better bounds on both the price of anarchy and the price of stability in this setting. The following two results cover this case. Again, we shall give a lower bound on the price of stability and a matching upper bound on the price of anarchy.

Proposition 6. For any two integers $n, m>1$, we have $\operatorname{PoS}(n, m) \geq 2-1 / n$ if $m \geq n, \operatorname{PoS}(n, m) \geq \frac{n+1}{n}$ if $n>m=2$, and $\operatorname{PoS}(n, m) \geq 2-\frac{1}{m-1}$ if $n>m>2$ for games with identical weights.

We shall prove the upper bounds by exploiting the primal-dual method developed in 24. Before doing this, we need some additional notation. Given two strategy profiles $\sigma$ and $\sigma^{*}$, denote as $\alpha\left(\sigma, \sigma^{*}\right)=\left|P\left(\sigma^{*}\right) \backslash P(\sigma)\right|$; moreover, for each $j \in M$, denote as $C_{j}\left(\sigma, \sigma^{*}\right)=\left\{i \in N: \sigma_{i}=\sigma_{i}^{*}=j\right\}$ the set of players selecting project $j$ in both $\sigma$ and $\sigma^{*}$ and as $O_{j}\left(\sigma, \sigma^{*}\right)=\left\{i \in N \backslash C_{j}\left(\sigma, \sigma^{*}\right): \sigma_{i}^{*}=j\right\}$ the set of players selecting project $j$ in $\sigma^{*}$ but not in $\sigma$. In the application of this method, we shall make use of the following technical lemma.

Lemma 1. Fix a game with identical weights. For each strategy profile $\sigma$ and social optimum $\sigma^{\prime}$, there exists a social optimum $\sigma^{*}$ such that (i) $P\left(\sigma^{*}\right)=P\left(\sigma^{\prime}\right)$ and (ii) for each $j \in P\left(\sigma^{*}\right) \cap P(\sigma),\left|C_{j}\left(\sigma, \sigma^{*}\right)\right| \geq L(\sigma, j)-\alpha\left(\sigma, \sigma^{*}\right)$.

Proof. Fix a strategy profile $\sigma$ and a social optimum $\sigma^{\prime}$ and, for the sake of simplicity, set $\alpha=\alpha\left(\sigma, \sigma^{\prime}\right)$. Our aim is to slightly modify $\sigma$ so as to obtain a social optimum $\sigma^{*}$ mimicking the assignment of players to projects realized in $\sigma$ for as much as possible. To do this, consider the following algorithm operating in three steps.

At step 1, for each $j \in P\left(\sigma^{\prime}\right) \backslash P(\sigma)$, choose a unique player $o(j)$ such that $j \in S_{o(j)}$ and define $\sigma_{o(j)}^{*}=j$. Let $T_{1}$ be the set of players chosen at this step; clearly, $\left|T_{1}\right|=\alpha$. At step 2, for each $j \in P\left(\sigma^{\prime}\right) \cap P(\sigma)$, choose a unique player $o(j)$ in $N \backslash T_{1}$ such that $j \in S_{o(j)}$ and define $\sigma_{o(j)}^{*}=j$. Let $T_{2}$ be the set of players 
chosen at this step. At step 3, for each $i \notin T_{1} \cup T_{2}$, set $\sigma_{i}^{*}=\sigma_{i}$ if $\sigma_{i} \in P\left(\sigma^{\prime}\right) \cap P(\sigma)$ and $\sigma_{i}^{*}=j$ otherwise, where $j$ is an arbitrary project in $P\left(\sigma^{\prime}\right)$.

The existence of $\sigma^{\prime}$ implies that there exists a choice for $T_{1}$ and $T_{2}$ which guarantees that $P\left(\sigma^{*}\right)=P\left(\sigma^{\prime}\right)$. To show part (ii) of the claim, consider a project $j^{*} \in P(\sigma) \cap P\left(\sigma^{*}\right)$ such that $L\left(\sigma, j^{*}\right) \geq \alpha$ (if no such project exists, then the claim is trivially true). Let

$$
\beta=\left|\left\{j \in P\left(\sigma^{\prime}\right) \cap P(\sigma):\left\{i \in N \backslash T_{1}: \sigma_{i}=j\right\}=\emptyset\right\}\right|
$$

be the number of projects in $P\left(\sigma^{\prime}\right) \cap P(\sigma)$ that lost all of their users in $\sigma$ after step 1 of the algorithm. We have that step 1 selects at least $\beta$ players from $\beta$ different projects in $P(\sigma) \cap P\left(\sigma^{*}\right)$. This implies that $j^{*}$ loses at most $\alpha-\beta$ users after step 1. At step $2, j^{*}$ can lose at most other $\beta$ additional users for a total of $\alpha$ users. Hence, at least $L(\sigma, j)-\alpha$ players are assigned to $j^{*}$ in $\sigma^{*}$ at step 3 of the algorithm and this shows claim (ii).

Theorem 9. For any two integers $n, m>1$, we have $\operatorname{PoA}(n, m) \leq 2-1 / n$ if $m \geq n, \operatorname{PoA}(n, m) \leq \frac{n+1}{n}$ if $n>m=2$, and $\operatorname{PoA}(n, m) \leq 2-\frac{1}{m-1}$ if $n>m>2$ for games with identical weights.

Proof. Fix a pure Nash equilibrium $\sigma$ and a social optimum $\sigma^{*}$ and, for the sake of simplicity, set $\alpha=\alpha\left(\sigma, \sigma^{*}\right)$. By Lemma 1, we can assume without loss of generality that, for each $j \in P\left(\sigma^{*}\right) \cap P(\sigma),\left|C_{j}\left(\sigma, \sigma^{*}\right)\right| \geq L(\sigma, j)-\alpha$. We assume $\alpha \geq 1$ as, otherwise, the price of anarchy is trivially equal to 1 . By applying the primal-dual method, we get that the inverse of the optimal solution of the following linear program provides an upper bound on $\operatorname{PoA}(n, m)$ :

$$
\begin{array}{ll}
\min \sum_{j \in P(\sigma)} r_{j} & \\
\text { s.t. } & \\
\frac{r_{\sigma_{i}}}{L\left(\sigma, \sigma_{i}\right)}-\frac{r_{\sigma_{i}^{*}}}{L\left(\left(\sigma_{-i}, \sigma_{i}^{*}\right), \sigma_{i}^{*}\right)} \geq 0 & \forall i \in N, \\
\sum_{j \in P\left(\sigma^{*}\right)} r_{j}=1 & \forall j \in M \\
r_{j} \geq 0 &
\end{array}
$$

For a strategy profile $\tau$ and a project $j$, denote by $1_{j}(\tau)$ the indicator function that is equal to 1 if and only if $j \in P(\tau)$. The dual of the above linear program is the following (we associate variable $x_{i}$ with the first constraint for each $i \in N$ and variable $\gamma$ with the second one):

$$
\begin{array}{ll}
\max \gamma & \\
\text { s.t. } & \\
\sum_{i: \sigma_{i}=j} \frac{x_{i}}{L(\sigma, j)}-\sum_{i: \sigma_{j}^{*}} \frac{x_{i}}{L\left(\left(\sigma_{-i}, j\right), j\right)}+\gamma 1_{j}\left(\sigma^{*}\right) \leq 1_{j}(\sigma) & \forall j \in M, \\
x_{i} \geq 0 & \forall i \in N
\end{array}
$$

The inverse of the objective value of any feasible solution to this program provides an upper bound on $\operatorname{PoA}(n, m)$.

First of all, we observe that, for any dual solution such that $x_{i}=x$ for each $i \in N$ and $\gamma=x$, the dual constrain becomes:

$$
x\left(1_{j}(\sigma)-\frac{\left|C_{j}\left(\sigma, \sigma^{*}\right)\right|}{L(\sigma, j)}-\frac{\left|O_{j}\left(\sigma, \sigma^{*}\right)\right|}{L(\sigma, j)+1}+1_{j}\left(\sigma^{*}\right)\right) \leq 1_{j}(\sigma) .
$$


If $1_{j}\left(\sigma^{*}\right)=0$, (3) is satisfied as long as $x \leq 1$. If $1_{j}\left(\sigma^{*}\right)=1$ and $1_{j}(\sigma)=0$, which imply $\left|C_{j}\left(\sigma, \sigma^{*}\right)\right|=0,\left|O_{j}\left(\sigma, \sigma^{*}\right)\right| \geq 1$, and $L(\sigma, j)+1=1$, (3) is satisfied independently of the value of $x$. The case of $1_{j}\left(\sigma^{*}\right)=1$ and $1_{j}(\sigma)=1$ is then the only one which can cause a price of anarchy higher than 1 and we focus on this case in the remainder on the proof. Note that, in this case, we can always assume $\left|C_{j}\left(\sigma, \sigma^{*}\right)\right|+\left|O_{j}\left(\sigma, \sigma^{*}\right)\right| \geq 1$.

Consider the dual solution such that $x=\frac{n}{2 n-1}$. As $L(\sigma, j)+1 \leq n,(3)$ is satisfied. This proves a general upper bound of $2-1 / n$. However, for the case of $n>m$, better upper bounds can be derived. Note that, in this case, we have $1 \leq \alpha \leq m-1$.

Assume $\alpha \leq m-2$ and consider the dual solution such that $x=\frac{m-1}{2 m-3}$. If $L(\sigma, j) \leq \alpha$, the term within the parenthesis in the left-hand side of $(3)$ is at most $\frac{2 m-3}{m-1}$ and the constraint is satisfied. If $L(\sigma, j)>\alpha$, as $\left|C_{j}\left(\sigma, \sigma^{*}\right)\right| \geq$ $L(\sigma, j)-\alpha$, the term within the parenthesis in the left-hand side of (3) is at most $2-\frac{L(\sigma, j)-\alpha}{L(\sigma, j)}=\frac{L(\sigma, j)+\alpha}{L(\sigma, j)}$ which is maximized for $\alpha=m-2$ and $L(\sigma, j)=$ $\alpha+1=m-1$. Again, $\sqrt{3}$ is satisfied. This proves an upper bound of $2-\frac{1}{m-1}$. Note that this bound does not apply to the case of $m=2$, as $\alpha$ cannot be equal to $m-2$ in this case.

Assume now $\alpha=m-1$ and consider the dual solution such that $x=\frac{n}{n+m-1}$. The assumption $\alpha=m-1$ implies that there exists a unique project $j \in$ $P(\sigma) \cap P\left(\sigma^{*}\right)$ and so $L(\sigma, j)=n$ and $C_{j}\left(\sigma, \sigma^{*}\right)=n-m+1$. In this case, the term within the parenthesis in the left-hand side of $(3)$ is exactly $\frac{n+m-1}{n}$ and (3) is satisfied. This proves an upper bound of $\frac{n+m-1}{n}$.

As $2-\frac{1}{m-1} \geq \frac{n+m-1}{n}$ for $n>m>2$, the claimed upper bounds follow.

\section{Egalitarian Social Welfare}

So far we have considered the utilitarian social welfare $\mathrm{U}(\sigma):=\sum_{i \in N} u_{i}(\sigma)$. In this section we use the egalitarian social welfare $\mathrm{E}(\sigma):=\min _{i \in N} u_{i}(\sigma)$ (to be maximized). For this section we suppose adapted definitions of the PoA and the PoS which include $E$ instead of $U$. The motivation for considering $E$ instead of $\mathrm{U}$ is fairness among the players.

Proposition 7. For the egalitarian social welfare, the PoS of the PROJECT GAME is unbounded even with 4 players, 2 projects, universal weights and identical rewards.

Since $\mathrm{PoA} \geq \mathrm{PoS}$, the PoA of the PROJECT GAME is unbounded as well. One can be tempted to try to enforce a social optimum. However, unlike the utilitarian social welfare (see Proposition 3), the problem is intractable.

Proposition 8. It is $\mathbf{N P}$-hard to compute a strategy profile that maximizes the egalitarian social welfare of the PROJECT GAME even if there are two projects, identical rewards, and universal weights.

Nevertheless, we were able to identify a polynomial case. 
Proposition 9. Maximizing the egalitarian social welfare of the PROJECT GA$\mathrm{ME}$ can be done in polynomial time when the players have identical weights.

\section{Conclusion and Open Problems}

We introduced a new class of games sharing similarities with valid utility games, singleton congestion games, and hedonic games. We focused on existence, computational complexity and efficiency of pure Nash equilibria under a natural method for sharing the rewards of the projects that are realized.

Though the existence of a pure Nash equilibrium is showed for many important special cases, proving (or disproving) its existence in general is a challenging task. An interesting special case that is left open is when the number of projects is small (e.g. $m=2$ ). Other solution concepts (e.g. strong Nash equilibria) deserve attention.

Our upper bounds on PoA and PoS under the utilitarian social welfare never exceed 2, but it does not prevent to explore other sharing methods. Moreover, closing the gap shown in Theorem 7 is an intriguing open problem.

Regarding the computation of an optimal strategy profile with respect to the egalitarian social welfare, there is a gap between hard and polynomial cases (see Propositions 8 and 9). As a first step, it would be interesting to settle the complexity of the symmetric case. As the PoS is unbounded under the egalitarian social welfare, it is natural to ask if a different reward sharing method can provide better results.

\section{References}

1. Koutsoupias, E., Papadimitriou, C.H.: Worst-case equilibria. In: Proc. of the 16th Annual Symposium on Theoretical Aspects of Computer Science (STACS). (1999) 404-413

2. Anshelevich, E., Dasgupta, A., Kleinberg, J.M., Tardos, É., Wexler, T., Roughgarden, T.: The price of stability for network design with fair cost allocation. In: Proc. of the 45th Symposium on Foundations of Computer Science (FOCS). (2004) 295-304

3. Morris, S., Ui, T.: Best response equivalence. Games and Economic Behavior 49 (2004) 260-287

4. Vetta, A.: Nash equilibria in competitive societies, with applications to facility location, traffic routing and auctions. In: Proc. of the 43rd Symposium on Foundations of Computer Science (FOCS). (2002) 416-425

5. Augustine, J., Chen, N., Elkind, E., Fanelli, A., Gravin, N., Shiryaev, D.: Dynamics of profit-sharing games. Internet Mathematics 11 (2015) 1-22

6. Bachrach, Y., Syrgkanis, V., Vojnovic, M.: Incentives and efficiency in uncertain collaborative environments. In: Proc. of the 9th Conference on Web and Internet Economics (WINE). (2013) 26-39

7. Gollapudi, S., Kollias, K., Panigrahi, D., Pliatsika, V.: Profit sharing and efficiency in utility games. In: Proc. of the 25th Annual European Symposium on Algorithms (ESA). (2017) 43:1-43:14 
8. Goemans, M.X., Li, L., Mirrokni, V.S., Thottan, M.: Market sharing games applied to content distribution in ad hoc networks. IEEE Journal on Selected Areas in Communications 24 (2006) 1020 - 1033

9. Kleinberg, J.M., Oren, S.: Mechanisms for (mis)allocating scientific credit. In: Proc. of the 43rd ACM Symposium on Theory of Computing (STOC). (2011) $529-538$

10. Marden, J.R., Roughgarden, T.: Generalized efficiency bounds in distributed resource allocation. In: Proc. of the 49th IEEE Conference on Decision and Control (CDC). (2010) 2233-2238

11. Marden, J.R., Wierman, A.: Distributed welfare games. Operations Research 61 (2013) $155-168$

12. Rosenthal, R.: A class of games possessing pure-strategy Nash equilibria. International Journal of Game Theory 2 (1973) 65-67

13. Monderer, D., Shapley, L.S.: Potential games. Games and Economic Behavior 14 (1996) $124-143$

14. Ieong, S., McGrew, R., Nudelman, E., Shoham, Y., Sun, Q.: Fast and compact: A simple class of congestion games. In: Proc. of the 20th National Conference on Artificial Intelligence (AAAI). (2005) 489-494

15. Milchtaich, I.: Congestion games with player-specific payoff functions. Games and Economic Behavior 13 (1996) 111 - 124

16. Vöcking, B.: Selfish load balancing. In Nisan, N., Roughgarden, T., Tardos, E., Vazirani, V.V., eds.: Algorithmic Game Theory. Cambridge University Press, New York, NY, USA (2007) 517-542

17. Drèze, J.H., Greenberg, J.: Hedonic coalitions: Optimality and stability. Econometrica 48 (1980) 987-1003

18. Darmann, A., Elkind, E., Kurz, S., Lang, J., Schauer, J., Woeginger, G.J.: Group activity selection problem. In: Proc. of the 8th International Workshop on Internet and Network Economics (WINE). (2012) 156-169

19. Fotakis, D., Kontogiannis, S., Koutsoupias, E., Mavronicolas, M., Spirakis, P.: The structure and complexity of nash equilibria for a selfish routing game. In: Proc. of the 29th International Colloquium on Automata, Languages and Programming (ICALP). (2002) 123-134

20. Gairing, M., Lucking, T., Mavronicolas, M., Monien, B.: Computing nash equilibria for scheduling on restricted parallel links. In: Proc. of the 36th Annual ACM Symposium on Theory of Computing (STOC). (2004) 613-622

21. Fabrikant, A., Papadimitriou, C.H., Talwar, K.: The complexity of pure nash equilibria. In: Proc. of the 36th Annual ACM Symposium on Theory of Computing (STOC). (2004) 604-612

22. Gairing, M., Monien, B., Tiemann, K.: Routing (un-)splittable flow in games with player-specific linear latency functions. In: Proc. of the 33rd International Colloquium on Automata, Languages and Programming (ICALP). (2006) 501$-512$

23. Georgiou, C., Pavlides, T., Philippou, A.: Selfish routing in the presence of network uncertainty. Parallel Processing Letters 19 (2009) 141-157

24. Bilò, V.: A unifying tool for bounding the quality of non-cooperative solutions in weighted congestion games. Theory of Computing Systems 62 (2018) 1288 - 1317 